\title{
An Age-Structured Single-State Drug Initiation Model - Cycles of Drug Epidemics and Optimal Prevention Programs*
}

\author{
Christian Almeder ${ }^{\dagger}$ \\ Department of Operations Research and Systems Theory \\ Vienna University of Technology \\ Jonathan P. Caulkins \\ H. John Heinz III School of Public Policy \& Management \\ Carnegie Mellon University \\ Gustav Feichtinger, and Gernot Tragler \\ Department of Operations Research and Systems Theory \\ Vienna University of Technology
}

13th June 2002

*This research was partly financed by the Jubiläumsfonds of the Österreichische Nationalbank under contract No. 8466 (Optimal Control of Age-Structural Economic Models)

${ }^{\dagger}$ corresponding author, Argentinierstraße 8/119, A-1040 Vienna, Austria; email: calmeder@eos.tuwien.ac.at, fax: +43-1-58801-11999

$\ddagger 5000$ Forbes Ave., Pittsburgh, PA 15213; email: caulkins@cmu.edu 


\begin{abstract}
This paper introduces a model for drug initiation that extends traditional dynamic models by considering explicitly the age distribution of the users. On the basis of a 2-groups model in which the population is split into a user and a non-user group the advantage of a continuous age distribution is shown by considering more details and by yielding new results. Neglecting death rates reduces the model to a single state (1-group) descriptive model which can still simulate some of the complex behavior of drug epidemics such as repeated cycles. Furthermore, prevention programs - especially school-based programs - can be targeted to certain age classes. So in order to discover how best to allocate resources to prevention programs over different age classes we formulate and solve optimal control models.
\end{abstract}

Keywords: Nonlinear dynamic system, age-structured model, illicit drug, partial differential equation, optimal control. 


\section{Introduction}

Often models of drug initiation and drug use are based on the same principles as epidemiological models, because drug use is clearly contagious in the sense that use by some individuals affects the probability that others will use through multiple mechanisms. In a very literal sense, most users are introduced to drug use by a friend or relative; the more drug users there are, the more likely an individual is to be offered the drug (see Kaplan [14]). At a market level, the larger the market, the more diluted the enforcement risk, and the safer it is to try drugs (see Kleiman [17]). At a reputational level, experiences of others can be instrumental in shaping perceptions of the riskiness of drugs, and those perceptions in turn influence initiation. Indeed, the very fact that the mechanism of transmission does not involve physical contact or interaction means that the dynamics of contagion can be more complex and more interesting.

Looking at drug use and the process of initiation in more detail, it is clear that the decision of a non-user to start consumption depends strongly not only on the individual's immediate, personal social environment, but also on the overall reputation of a drug in society, e.g., as portrayed in movies or news media. That means, an individual might want to use drugs even if none of the individual's associates encourages that desire. And, conversely, an individual may fear drugs even if no one he or she knows has suffered harm from them.

Cycles or epidemics of greater and lesser drug use have been observed for licit and illicit drugs over extended periods of time. Behrens et al. [3, 2] 
formalized one possible explanation of these cycles. Newly initiated users are presumed to be light users, and light users promote further initiation through a contagion effect, creating a positive feedback cycle. Over time, some light users escalate to problematic or heavy use. Those heavy users then serve as a sort of negative advertisement for the drug, suppressing further initiation, until the memory of those heavy users decays, paving the way for a renewed cycle of initiation.

Here we explore another model of reputational feedback that by itself can account for explosive growth, a subsequent negative feedback, and, at least for some parameter values, cycling. The key extension is the notion that reputation feeds back differently to, and perhaps even from, people of different ages. The Behrens et al. papers do not model the individual's ages explicitly, so there is just one universal reputation level and initiation is aggregated, not age specific. Here we allow for the possibility that a given substance could have a positive reputation among some age groups (e.g., the young) at the same time it has a negative reputation among others (e.g., their parents). Furthermore this difference is not generated exogenously, e.g., by postulating that youth and their parents read different books or watch different television shows. Rather, it emerges from plausible hypotheses concerning how experience with drugs by one age or birth cohort might influence the attractiveness of that substance to people in other cohorts. That is, it is the age-specific pattern of drug experience that generate the age-specific reputations and associated influences on initiation. It is, in that sense, a model of how intergenerational interaction can influence consumption dynamics as well as a model of drug use per se. 
Note there is evidence of both short-term and long-term cycling in drug use. E.g., contemporary variation in US marijuana initiation [13], Australian heroin initiation [15], and Italian heroin overdose deaths [24] shows some evidence of oscillations with a period of 5-12 years. Over the long term, there have been several distinct peaks in US alcohol consumption over the last 150 years and two peaks in heroin and cocaine consumption - one in the early 1900s and one around 1990 [25, 21, 22]. Similar cycles are observed in other countries, including for alcohol in Australia [9] and stimulants in Japan [28]. The intergenerational dynamics investigated in this paper would seem more likely to bear on those long-wave cycles than on oscillation with a period of less than 20 years.

The Behrens et al. model in which heavy or addicted use dampens initiation is most appealing for so-called hard drugs such as cocaine or heroin. This model, with its emphasis on social interaction, may be more appropriate for other drugs such as marijuana or cigarettes. Since it does not rely on addiction mechanisms, it might even be useful for modeling other consumer behaviors, such as preferences for styles or brands of clothing.

A simple way to introduce age is to split the population into different age groups. This leads to compartment models but with a large number of population groups (see Swan [27]). Analysis of such models is difficult, and it is necessary to increase the number of age groups in order to get a better approximation. A more general and more elegant method is to include age as a second parameter in addition to time. So a continuous age distribution of the population can be fully taken into consideration, and the model description and analysis is independent of the number of age groups 
for which data are available. The analysis of such a model becomes in some way easier, because there are fewer groups to consider, but in some sense also more complicated, because this method leads to a system of partial differential equations - a further development of the so-called McKendrick equation (see Keyfitz and Keyfitz [16]).

In epidemiology, several papers on age-structured models are available. Murray [20, pp. 640-650] describes a simple SI (susceptible - infected) model with an age-dependent infectiousness, which can be solved analytically. Busenberg et al. [5] and Iannelli et al. [12] investigate the global behavior and threshold properties of age-structured SIS (susceptible - infected - susceptible) models, and Müller [18, 19] concentrates on optimal vaccination patterns for age-structured SIR (susceptible - infected - recovered) models, but without time-dependence of the control.

Although the epidemiological models are very similar to the model described in this paper, there are some essential features in drug initiation, which do not allow one to transfer blindly the results to the initiation models.

This paper is organized as follows. We start with the formulation of the descriptive model in section 2, followed by a description of the analytical and numerical methods necessary for simulation and optimizing experiments in section 3. In section 4 results are discussed for both simulation of the uncontrolled model and the design of optimal prevention programs for a slightly simplified model. The paper ends with general conclusions and an outlook to future work in section 5 . 


\section{Model Formulation}

\subsection{Development of Model Equations}

We consider a population divided into two groups: non-users and users (or drug consumers). Neglecting any death or migration and assuming constant birth cohort size, the sum of the non-user and the user populations is always constant, so it is sufficient to consider only one state (e.g., the non-user population).

Furthermore, only the flow from the non-user state into the user state is taken into consideration, which means that once an individual has consumed drugs, he or she will never stop being identified as a drug consumer. So the user population does not represent the number of current consumers (pastyear prevalence), but rather the number of people who have ever used drugs (lifetime prevalence).

The following equation describes the non-users' dynamics in terms of $P(t, a)$ - the number or population of non-users aged $a$ at time $t$ - and $\mu(t, a)$ - the initiation rate:

$$
P_{t}+P_{a}=-\mu(t, a) P(t, a) .
$$

In essence this so-called McKendrick equation (Keyfitz and Keyfitz [16]) simply says that the rate of change of the non-users equals (minus) the per capita initiation rate times the number of non-users, but it does this for a continuous distribution of ages $(a)$, not just an aggregated pool. The initiatial conditions give the number of non-users of various ages at time $t=0$, and the boundary condition specifies how many people of age $a=0$ are born at 
each time, i.e.

$$
\begin{aligned}
& P(0, a)=P_{0}(a), \\
& P(t, 0)=k,
\end{aligned}
$$

for a finite planning horizon $t \in[0, T]$ and age range $a \in[0, \omega]$, where $P_{0}(a)$ is the initial population distribution at $t=0$, and $k$ is a fixed birth cohort size. (We choose $k=1$ and so interpret $P(t, a)$ as the proportion of non-users.)

The rate of initiation $\mu(t, a)$ is assumed to be the product of three different factors:

1. a basic, age-specific initiation rate $\bar{\mu}(a)$ representing the probability that a non-user starts drug consumption without any influence from others or prevention;

2. the influence $\Phi(R(t, a))$ of the reputation of the drug $R(t, a)$ on the initiation of a non-user of age $a$; and

3. a prevention factor $\Psi(w(t, a))$ incorporating the effects of age-specific prevention programs $w(t, a)$ on the initiation rate.

Summarizing, we have

$$
\mu(t, a)=\bar{\mu}(a) \Phi(R(t, a)) \Psi(w(t, a)) .
$$

The function $R(t, a)$ represents the reputation of the drug at time $t$ as perceived by a non-user of age $a$. Discussions of reputational feedback sometimes distinguish between the effects of society-wide reputation of the drug, as portrayed in news media and movies, and an individual reputation, which explicitly reflects reputational interaction across age groups. We include both 
and denote them by $R_{\text {soc }}(t, a)$ and $R_{\text {ind }}(t, a)$, respectively. We do not imagine that the societal-wide reputation affects all ages equally. E.g., the young may be more impressionable than adults. But the effects of the societal-wide reputation are separable into an age-specific but time-invariant vulnerability to reputation effects and an age-invariant but state (and hence time) dependent society-wide reputation. The society-wide and individual reputations are assumed to be driven by the weighted averages of the levels of drug (non)-use relative to some benchmark level, i.e.

$$
\begin{aligned}
& R_{\mathrm{soc}}(t, a)=m_{1}(a) \int_{0}^{\omega} m_{2}\left(a^{\prime}\right)\left(i_{u}-\frac{P\left(t, a^{\prime}\right)}{k}\right) d a^{\prime} \\
& R_{\text {ind }}(t, a)=\quad \int_{0}^{\omega} m_{3}\left(a, a^{\prime}\right)\left(i_{u}-\frac{P\left(t, a^{\prime}\right)}{k}\right) d a^{\prime}
\end{aligned}
$$

where the functions and constants have the following meaning:

$m_{1}(a)$ measures the degree of influence the overall reputation of the drug has on a non-user of age $a$. Usually it is assumed that it decreases with age, which means that older people are less influenced by popular opinions than younger people are.

$m_{2}\left(a^{\prime}\right)$ describes how influential people of age $a^{\prime}$ are for the overall reputation of the drug. It should be large for age groups that are opinion leaders. When it comes to consumption trends, whether of legal or illegal products, behavior of young people is generally considered to be more influential.

$m_{3}\left(a, a^{\prime}\right)$ indicates the direct influence of users and non-users of age $a^{\prime}$ on a non-user of age $a$. This influence depends primarily on the age difference. Especially for young non-users (who have the highest underlying 
proclivity to initiate into drug use) it is assumed, that persons who are of the same age or a little bit older are role models, and therefore their influence is very high. On the other hand, consumption behavior of persons who are a generation older have less an impact and may ever evoke a contrarian response.

$i_{u}$ reflects the level of activity that differentiates stigmatized and normative behavior. If $i_{u}=1$, then use contributes positively to the reputation term, regardless of the level of use. If $i_{u}=0.9$ then when drug use is uncommon (below 10\%), that use is stigmatized and contributes negatively to the drug's reputation.

The total reputation is a combination of the both reputation terms which leads to

$$
R(t, a)=\int_{0}^{\omega} m\left(a, a^{\prime}\right)\left(i_{u}-\frac{P\left(t, a^{\prime}\right)}{k}\right) d a^{\prime}
$$

with

$$
m\left(a, a^{\prime}\right)=i_{\text {ind }} m_{3}\left(a, a^{\prime}\right)+\left(1-i_{\text {ind }}\right) m_{1}(a) m_{2}\left(a^{\prime}\right)
$$

representing the weighted sum of individual and societal reputation, where $i_{\text {ind }} \in[0,1]$ measures the influence of the individual reputation and $\left(1-i_{\text {ind }}\right)$ that of the society-wide reputation.

The function $\Phi($.$) transforms the reputation into an effect on initiation.$ Presumably it is a non-negative monotonically increasing function with a floor and ceiling. That is, no matter how bad a drug's reputation some people will still initiate, and no matter how good the reputation some people will still abstain. By definition $\bar{\mu}(a)$ reflects initiation with a neutral reputation 
SO

$$
\Phi(R)= \begin{cases}d \in[0,1] & \text { for } R \rightarrow-\infty \\ 1 & \text { for } R=0 \\ e \in[1, \infty) & \text { for } R \rightarrow \infty\end{cases}
$$

meaning that zero reputation has no impact on the initiation, a very negative reputation reduces the initiation by $(1-d) \cdot 100 \%$, while a very positive reputation increases the rate by $(e-1) \cdot 100 \%$. Data clearly correlate drug's reputation with initiation, but to the best of our knowledge no one has postulated a specific functional form, so that is deferred until later in this paper.

Following Behrens et al. [1] the function $\Psi($.$) describing the influence of$ prevention on initiation is assumed to be of the form

$$
\Psi(w(t, a))=(1-c) e^{-\varepsilon w(t, a)}+c
$$

where $(1-c) \in(0,1)$ measures the maximal proportionate reduction in initiation and $\varepsilon$ reflects the efficiency of prevention spending. In essence this form simply says the more that is spent on prevention, the lower initiation is, but there are diminishing returns modeled as an exponential decay in marginal effectiveness.

In order to evaluate the effectiveness of a prevention program it is necessary to define an objective which should be minimized (or maximized). In our case we have chosen the discounted total social costs as the sum of costs induced by drug consumption and by prevention expenditures,

$$
J=\int_{0}^{T} e^{-r t} \int_{0}^{\omega}(\rho(a)(k-P(t, a))+w(t, a)) d a d t \longrightarrow \min _{w(t, a)}
$$


where $\rho(a)$ represents the age specific average annual costs of someone who has ever consumed drugs. Of course, other forms of the objective are possible, which, e.g., incorporate the initiation directly.

\subsection{Specification of Functions and Parameter Values}

Four functions remain to be specified. They are discussed separately because they are most likel to vary from drug to drug or from country to country. Those we choose here are meet to plausible for

- $\bar{\mu}(a)$ : Three different basic initiation functions are used. The first is constant. The second is a step function with a constant positive initiation below age 25 and zero initiation for older people. The third matches data for marijuana initiation in the US. It has a sharp peak around the age of 16 years and is nearly zero outside the interval [10,25] (see figure 1). The third is the most realistic, but contrasting its results with those for the first two stylized initiation functions yields insight into the qualitative character of the model behavior.

[Figure 1 about here.]

- $m\left(a, a^{\prime}\right)$ describes the overall influence of the users of age $a^{\prime}$ on non-users of age $a$. For $m_{3}\left(a, a^{\prime}\right)$ a form is used where the influence of people who are 2 years older is maximal, while it decreases to a negative value for users, who are more than 12 years older or more than 8 years younger. With increasing age difference the influence stays negative, but converges to zero. Also with increasing age of the non-user the absolute 
value $\left|m_{3}\left(a, a^{\prime}\right)\right|$ decreases. The societal part of the influence, $m_{2}\left(a^{\prime}\right)$, is chosen so that the age range from 20 to 30 years is the age group with the largest influence on the overall reputation. Following the idea that younger people are more influenced by media the function $m_{1}(a)$ is monotonically decreasing. The implication of these assumptions is depicted in figure 2 .

[Figure 2 about here.]

- $\Phi($.$) measures the influence of reputation on the initiation rate. To$ satisfy equation (2.8) it is set to

$$
\Phi(R)=d_{1}+\frac{1}{d_{2} \pi} \arctan \left(d_{3} * R\right)
$$

where $d_{1}$ (default value: 1 ) and $d_{2}$ (default value: 1 ) determine the decrease and increase of the initiation rate due to the drug's reputation, and $d_{3}$ represents the rate of response to changes of the reputation.

[Figure 3 about here.]

- $\rho(a)$ : For marijuana, careers of use are shorter than for alcohol or cigarettes, so it makes sense to iassess an age-specific penalty with initiation, rather than thinking of a cost per unit time on an ongoing basis with people who have ever used (and may have lnog since quit). We do this by setting $\rho(a)$ equal to a constant, which is equivalent to assuming that the lifetime costs of drug use decrease exponentially with the age of initiation. When $\rho(a)$ is constant, the lifetime costs of 
someone initiating at age $\tau$ are

$$
\int_{\tau}^{\omega} e^{-r a} \rho d a=\frac{\rho}{\tau}\left(e^{-r \tau}-e^{-r \omega}\right) .
$$

Figure 4 compares this social function with estimates of lifetime consumption of marijuana and cocaine [6]) with all three plotted as a function of the age at marijuana initiation. The essential observation is that average lifetime consumption declines sharply with age of initiation, and setting $\rho(a)$ to a constant generates a cost function that reflects this decline.

[Figure 4 about here.]

The following further parameters were used in the simulation experiments:

$$
\begin{array}{ll}
\text { age }=10-60 & \text { (age range) } \\
P_{0}(a) \equiv 1 \quad \text { (initial population distribution) } \\
i_{\text {loc }}=0.75 \quad \text { (influence of local reputation) } \\
i_{u}=0.9 \quad \text { (user }- \text { non-user relation for the reputation) }
\end{array}
$$

Due to the complex nature of its equations, the model cannot be solved analytically. Indeed even numerical analysis is non-trivial and requires development of some algorithms and properties. This is done in the next section. Readers uninterested in this mathematics are encouraged to proceed directly to section 4 which describes the results of simulation experiments on different data sets and calculation of optimal prevention programs for a simplified model. 


\section{Numerical and Optimization Methods}

\subsection{Applying the Method of Lines}

Before applying a numerical algorithm to our problem it is necessary to make some numerical transformations. Using the method of lines (cf. Schiesser [26]) the partial differential equation (2.1) is rewritten as a system of ordinary differential equations by discretizing the age and substituting the partial derivatives w.r.t. $a$ by finite approximations. Hence, instead of a continuous age variable $a$ we have now a finite series of age classes $\left(a_{i}\right)_{i=0 . . N_{a}}$.

Formally this separation into age classes leads to a compartment model. Note, however, that here we are describing an algorithmic solution method, which should be detached from the modeling process. The formulation of the model uses a continuous age distribution; for numerical reasons it is necessary to change to a discrete distribution. The advantage of moving this discretization step from the modeling phase into the solution algorithm phase is that the number of age classes can be chosen solely with respect to considerations of computational precision, and without regard to availability of data or issues of model interpretation.

The usual way to solve these equations would be the method of characteristics which reduces the partial differential equation (PDE) to ordinary differential equations (DOE) along the family of lines $t=a+c$. This avoids discretization errors in this first step. But the calculation of integrals with respect to age - as they occur in the reputation term - requires an interpolation between the grid points or a synchronous integration of the ODE equations with a certain shift depending on the starting point. So in this 
case discretization along the age dimension is preferred.

Equation (2.1) then becomes

$$
\begin{aligned}
& P_{a_{0}}(t)=k, \\
& P_{a_{i}}^{\prime}(t)=-\Delta_{a}\left[P_{a_{i}}(t)\right]-\mu_{a_{i}}(t) P_{a_{i}}(t) \quad i=1, \ldots, N_{a},
\end{aligned}
$$

where $\boldsymbol{\Delta}_{\boldsymbol{a}}$ denotes an appropriate finite difference operator w.r.t. $a$.

The initial condition of equation (2.2) leads to initial conditions for the ODEs:

$$
P_{a_{i}}(0)=P_{0}\left(a_{i}\right) \quad i=1, \ldots, N-a .
$$

The initiation rate is transformed analogously to individual initiation rates for each age class $a_{i}$

$$
\mu_{a_{i}}(t)=\mu_{a_{i}} \Phi\left(\boldsymbol{\Omega}_{\boldsymbol{a}}\left[m_{a_{i}, a_{j}}\left(1-\frac{P_{a_{j}}(t)}{k}\right)\right]\right) \Psi\left(w_{a_{0}}(t), \ldots, w_{a_{N_{a}}}(t)\right),
$$

where $\boldsymbol{\Omega}_{\boldsymbol{a}}$ is the approximation function for the integral w.r.t. age. The Newton-Cotes formula (e.g. trapezoid formula for first-order or the Simpson formula for third order) is suitable in this case.

It is important to notice that the PDE (2.1) is hyperbolic, which means that discontinuities are propagated with time along the $a$-axis. This must be taken into consideration when choosing an appropriate finite difference operator. As the propagation is directed towards the positive $a$-axis, the simplest operator is the first-order two-point upwind approximation. Suitable operators of higher order can be derived from the Taylor series. Schiesser [26] recommends using a combination of upwind and centered approximations in order to avoid artificial oscillations caused by discontinuous initial or boundary conditions. At the boundaries different approximations are used in order to avoid points outside the grid. 


\subsection{Necessary Optimality Conditions}

In this section the maximum principle is applied to two simplified versions of the optimal control model induced by minimizing the objective (2.10). Both have modified reputation terms, because existing methods described in literature are not general enough to cover the full model.

The underlying idea of the maximum principle is to calculate the optimum of the objective function under certain conditions coming from the model equations. So generally speaking a Langrangian function is defined and maximized by including multipliers, which are called costate or adjoint variables [11].

\section{Interaction only with individuals of the same age}

In this first case we take the control model (2.1)-(2.9) and (2.10), but replace the interaction between users and non-users of different ages with interaction between individuals only of the same age. So the integral in (2.6) vanishes and we have a new initiation rate of the form

$$
\mu(t, a)=\bar{\mu}(a) \Theta(P(t, a)) \Psi(w(t, a))
$$

where $\Theta(P(t, a))$ represents the reputation of the drug as it is transmitted by $a$-year old users and non-users to non-users of the same age.

The following theorem describes the necessary conditions of this simplified problem. (From now on we write the function arguments only if omitting them would cause misinterpretation.)

Theorem 1. If $w^{*}(t, a)$ is the optimal control for the dynamic system described through (2.1), (2.2), (2.9), (2.10), and (3.4), then there exists func- 
tions $P^{*}(t, a)$ defined through (2.1), (2.2), (2.9), (3.4) and $q^{*}(t, a)$ defined through

$$
q_{t}^{*}+q_{a}^{*}=-\rho+r q^{*}+\bar{\mu} \Psi\left(w^{*}\right)\left(P^{*} \Theta^{\prime}\left(P^{*}\right)+\Theta\left(P^{*}\right)\right) q^{*}
$$

together with

$$
\begin{array}{ll}
q^{*}\left(t, \omega_{a}\right)=0 & \text { for } 0 \leq t \leq T \\
q^{*}(T, a)=0 & \text { for } 0 \leq a \leq \omega_{a}
\end{array}
$$

and

$$
w^{*}=\left\{\begin{array}{l}
0 \quad \text { for }(1-c) \varepsilon \bar{\mu} q^{*} P^{*} \Theta\left(P^{*}\right) \leq 1 \\
\frac{1}{\varepsilon} \ln \left((1-c) \varepsilon \bar{\mu} q^{*} P^{*} \Theta\left(P^{*}\right)\right) \quad \text { otherwise }
\end{array}\right.
$$

which hold for $(t, a) \in[0, T] \times[0, \omega]$.

The proof of this theorem can be found in appendix A.1.

The terms of the adjoint equation (3.5) can be interpreted in our drug initiation context. The adjoint variable $q^{*}(t, a)$ can be regarded as the marginal value at time $t$ of the non-user population $P^{*}(t, a)$ of age $a$. So the effect of an additional non-user in the next time step can be split up term for term:

1. $q_{t}^{*}+q_{a}^{*}$ is the change of valuation of a non-user. It is equal to the sum of the following terms.

2. $-\rho+r q^{*}$ are the effects due to the social costs $(-\rho)$ and the discount rate $\left(r q^{*}\right)$.

3. $\bar{\mu} \Psi\left(w^{*}\right) \Theta\left(P^{*}\right) q^{*}$ is the effect of the additional initiation $\bar{\mu} \Psi\left(w^{*}\right) \Theta\left(P^{*}\right)$ valued with $q^{*}$, because an additional non-user is also a potential new user. 
4. $\bar{\mu} \Psi\left(w^{*}\right) \Theta^{\prime}\left(P^{*}\right) P^{*} q^{*}$ is the effect of the change of the initiation rate due to the additional non-user weighed with $q^{*}$.

\section{Society-wide reputation only $\left(i_{\text {ind }}=0\right)$}

Returning back to the original problem of (2.1)-(2.9), (2.10), but setting $i_{\text {ind }}=0$, means that we consider only the society-wide reputation $R_{\text {soc }}$. So we have a simplified reputation function:

$$
R_{\mathrm{soc}}(t, a)=m_{1}(a) \int_{0}^{\omega} m_{2}\left(a^{\prime}\right)\left(i_{u}-\frac{P\left(t, a^{\prime}\right)}{k}\right) d a^{\prime},
$$

and as initiation rate

$$
\mu(t, a)=\bar{\mu}(a) \Phi\left(R_{\mathrm{soc}}(t, a)\right) \Psi(w(t, a))
$$

Now we need a new method in order to apply the maximum principle, which is described in the next theorem.

Theorem 2. If $w^{*}(t, a)$ is the optimal control for the dynamic system described through (2.1), (2.2), (2.9), (2.10), (3.8), and (3.9), then there exists functions $P^{*}(t, a)$ and $R_{\text {soc }}^{*}(t, a)$ defined through (2.2), (2.9), (3.8), and (3.9) and $q^{*}(t, a)$ and $\theta^{*}(t)$ defined through

$$
\begin{aligned}
q_{t}^{*}+q_{a}^{*} & =-\rho+r q^{*}+\bar{\mu} \Phi\left(R_{s o c}^{*}\right) \Psi\left(w^{*}\right) q^{*}+\frac{m_{2}}{k} \theta \\
\theta(t) & =-\int_{0}^{\omega} \bar{\mu}(a) \Phi^{\prime}\left(R_{s o c}^{*}(t, a)\right) m_{1}(a) \Psi\left(w^{*}(t, a)\right) q^{*}(t, a) P^{*}(t, a) d a
\end{aligned}
$$

together with

$$
\begin{array}{ll}
q^{*}\left(t, \omega_{a}\right)=0 & \text { for } 0 \leq t \leq T \\
q^{*}(T, a)=0 & \text { for } 0 \leq a \leq \omega_{a}
\end{array}
$$


and

$$
w^{*}=\left\{\begin{array}{l}
0 \quad \text { for }(1-c) \varepsilon \bar{\mu} q^{*} P^{*} \Phi\left(R_{\text {soc }}^{*}\right) \leq 1 \\
\frac{1}{\varepsilon} \ln \left((1-c) \varepsilon \bar{\mu} q^{*} P^{*} \Phi\left(R_{\text {soc }}^{*}\right)\right) \quad \text { otherwise }
\end{array}\right.
$$

which hold for $(t, a) \in[0, T] \times[0, \omega]$.

The proof of this theorem can be found in appendix A.2.

The interpretation of the necessary conditions is pretty much the same as in the previous case. The integral term here represents the effects of the change of the initiation rate, due to the change of the reputation.

To get a numerical approximation of the solution of either form of the optimal control model, the model equations and the necessary optimality conditions have to be solved simultaneously. The Method of Lines is applied to the adjoint system as well, but because of the terminal conditions of the costate variables this method results in a system of ODEs where one half of the equations (originating from the model equations) have initial conditions and the second half (originating from the adjoint equations) have terminal conditions. So what we now have is a classical boundary value problem (BVP). Due to the structure of the problem shooting methods which are very fast and memory-saving cannot be applied. Hence, a collocation method is used, which essentially discretizes and transforms the system into a nonlinear equation system which is solved by a Newton method. 


\section{Results of Numerical Experiments}

\subsection{Results with the Uncontrolled Model}

We begin by considering what happens in the absence of prevention by studying the uncontrolled dynamics of our model. A central finding is that the system oscillates, i.e., there are recurring peaks of use. We initialize the model with everyone being a non-user $\left(P_{0}(a) \equiv 1\right)$ so results for the first peak represent the spread of a new drug (such as marijuana). In the longer run, the influence of the initial values declines, and the results represent patterns of use for a traditional drug (such as alcohol is today or marijuana may be in the future).

With an initiation function $\bar{\mu}(a)$ based on US marijuana data the results show that the system converges either to a limit cycle (as in figure 5), if changes in reputation lead to large changes in initiation (e.g. $d_{3}=3$ ), or to a stable equilibrium (figure 6), if initiation is less responsive to changes in reputation (e.g. $\left.d_{3}=0.5\right)$. In either case the transient behavior includes sharp variations in prevalence. This implies that for a new drug (all of the illicit drugs are relatively new), a history of oscillations in use does not necessarily mean that such oscillations will persist over the long run - as seems to be the case for alcohol in at least some societies [9, 25]. Interestingly the period of the limit cycles (about 70 years) is fairly close to the period of oscillations in alcohol use observed in the US and Australia and the gap between the first peak in US cocaine and heroin use (early 1900's) and the second (roughly 1990). 
[Figure 5 about here.]

[Figure 6 about here.]

The behavior of the solution depends not only on the steepness of the reputation function (see figure 3), but also on the form of the basic initiation rate $\bar{\mu}(a)$. In order to get cycles it is necessary to have some age groups with high initiation rates, and others with very low or zero basic initiation. If $\bar{\mu}(a)$ is constant for the whole age range, then the system always converges to an equilibrium. But if a simple step function is used (see figure 1), it is again possible to have cycles (cf. table 1).

The explanation for this lies in the form of the influence function $m\left(a, a^{\prime}\right)$. The negative influence of older users on young non-users can block the initiation of young people if the group of older users is large enough (see figure 2). However, if baseline initiation rates are high for older non-users, whose behavior is less sensitive to reputation effects, the number of users in that birth cohort will eventually increase, preventing that birth cohort from having a low number of users as it ages, and cycles will only emerge if some birth cohorts never have high initiation rates. On the other hand if baseline initiation rates for older individuals are low, then a cohort whose initiation is suppressed at younger ages will never use the drug in large numbers and, hence, will not be able to suppress youthful use by the next generation when they are older,so a new wave of users is created. If the age range in which the initiation is high shrinks, the amplitude of the resulting waves gets very large. Initiation of drug use and, many deviant behaviors, is indeed highly concentrated within a narrow age range. 
[Table 1 about here.]

\subsection{Results with Static Prevention Strategies}

The last four lines illustrate the effects of two different (static) strategies for prevention spending that do not evolve over time. I.e., $w(t, a)=w(a)$. The step strategy spends the same amount on all people between the ages of 10 and 25. The init" strategy matches prevention spending by age to the age distribution of baseline initiation. The different prevention programs reduce the amplitude of the oscillations only marginally, but the number of users as a whole is reduced enough to reduce the total social cost (last column). The results suggest that prevention is more effective at reducing social costs when drug use approaches an equilibrium rather than oscillating indefinitely, and it is more effective when it is matched closely to the age distribution of baseline initiation. Therefore it is of interest to investigate further the influence of prevention and how to optimize it, which we do next.

\subsection{Optimal Prevention Strategies}

We discuss next some numerical results for theorem 2. The results for this simpler model used in theorem 1 do not differ very significantly from those presented below, because due to our parameter choice for these experiments the influence of the reputation term on the model dynamics is low.

To solve the initial-boundary value problem described through the model equations and the necessary conditions of theorem 2 again the method of lines is used to transform it into an ordinary boundary value problem by 
discretizing the age. Furthermore, a finite difference method of the $N A G$ Fortran Library was used to solve those systems of ODEs.

With some exceptions the same parameters as for the simulation experiments described at the beginning of this section are used. Those exceptions are:

$$
\begin{array}{ll}
P_{0}(a)=\text { equil. } & \text { (initial values are set equal to the equilibrium) } \\
i_{\text {ind }}=0 & \text { (due to the requirements of theorem } 2) \\
d_{3}=3 & \text { (high response to changes of the drug's reputation) } \\
\bar{\mu}(a)=\text { marij./step } & \text { (two variants of the basic initiation rate, cf. table } 1)
\end{array}
$$

Due to the fact that only the social-wide reputation $R_{\text {soc }}(t, a)\left(i_{\text {ind }}=0\right)$ is taken into consideration, the uncontrolled system (without any prevention) converges always to an equilibrium. This equilibrium is chosen as the initial situation for the controlled model investigations.

The results of the numerical experiments are unspectacular, because the effectiveness of prevention in absolute terms is low $[7,6]$, i.e. the parameter $\varepsilon$ in equation (2.9) is small and $c=0.84$, which provokes a maximal possible reduction of the initiation rate of $16 \%$. Nevertheless the application of prevention reduces both the number of users and the total social costs. In the case of the marijuana data, the optimal prevention program reduces the total social cost by $2 \%$ while the number of users can be decreased by about $5 \%$ (cf. table 2).

[Table 2 about here.] 
Because prevention is modeled as affecting behavior immediatly, not with a lag, the optimal prevention expenditure follows more or less the basic initiation rate. Since the controlled model converges to an equilibrium, the optimal control does not vary much over time. Initially prevention spending changes a little bit in order to move the system from the uncontrolled to the controlled equilibrium. Then it stabilizes. The apparent large changes beginning around $t=120$ are an artifact of the finite planning horizon $(t=200$ years) and the absence of a salvage value function. They should be ignored. Indeed, the principal reason for solving the planning problem over such a large horizon is simply to prevent such artificial edge effects from contaminating the results for earlier years which are of genuine interest. The long time horizon of 200 years is necessary to find the optimal prevention in a stabilized situation. The reputational feedback lengthen the time periods where initial or terminal conditions are effective (see figures 7 and 8 for further details).

[Figure 7 about here.]

[Figure 8 about here.]

Figure 8 shows that it is not optimal to target prevention programs to people over 20 although there is some probability of initiating after that age. Either this probability is to small or people at that age are not so easily influenced and therefore the reputational effect of the drug is less potent.

If the optimal control problem is started with initial conditions reflecting no use at time 0 then the results for relative spending by age are similar spend the most on prevention for people in the ages with the highest baseline 
initiation rates — but the total amount of prevention spending over time oscillates along with levels of use (or non-use). Varying control over time can be challenging, given bureaucratic inertia, but this may be less problematic for long-wave variation than higher frequency variation in control efforts. It has been observed that alcohol control efforts have ebbed and flowed over time much as alcohol use itself has [10].

\section{Conclusions}

Introducing age-specific aspects to drug initiation models allows one to develop more realistic models that give insights into the principles of drug epidemics and their control. Even the simple model presented in this paper highlights an important contribution of age-structured models: the observed complex dynamics of cycles of drug use can be simulated using a model that does not depend on users escalating to a state of heavy or addicted use as in Behrens et al. [1]. Hence this model is a more plausible candidate for explaining oscillations in use of substances such as marijuana that do not generate adverse consequences that are as dramatic as for drugs such as cocaine or heroin.

The behavior of the solution (ongoing cycles or damped oscillations approaching an equilibrium) depends on the type of age-specific feedback. To gain similar results with models that do not differentiate by age a larger number of groups would be necessary.

In particular, the age-specific concept allows one to incorporate an agespecific reputation effect (feedback from number of users on the initiation 
rate), which depends on how much influence a user of age $a^{\prime}$ has on a nonuser of age $a$, and whether this influence is positive or negative.

Another advantage of age-specificity is the ability to investigate agestructured strategies for prevention programs and to optimize them. The results of the control models investigated here are unsurprising. Essentially they recommend having the age distribution of prevention spending closely mirror the age distribution of the baseline initiation. To some extent this is because the control model discussed in this paper considers only an instantaneous effect of prevention on the current initiation rate with no persistent impact. Developing models considering other types of prevention effects is straightforward. Solving them is a challenge because they demand extensions of the existing maximum principles, but they represent a potentially fruitful area for further research and an instance in which a practical managerial problem may drive the development of basic mathematical and economic methods.

To analyze age-specific models with richer structures, the next steps are the formulation of an extended maximum principle for the full reputation term and the introduction of more age-specific details. For instance, the efficiency and maximal impact of prevention on initiation ( $\varepsilon$ and $c$ in equation (2.9)) could be regarded as age-specific, so that prevention is more effective in younger age classes. Likewise one could imagine models that distinguish different types of users or introduce duration of use as a third parameter, because some aspects of drug initiation depend on how long an individual has been in his/her current group. For instance, the probability of an individual moving from light to heavy use may be related to the duration of his/her 
drug consumption.

In summary, the age-specific model presented in this paper generates some insights into the dynamics of drug epidemics that models neglecting age-specificity cannot, and formulating an associated optimal control model is a step towards increasing the effects of prevention programs by improving their targeting. Nevertheless, the price paid for including age-specifity was extreme simplification on other dimensions, so this paper is just an initial step down the path of age-specific modeling of drug epidemics, and further works would be valuable.

\section{A Proofs}

\section{A.1 Proof for Theorem 1}

The maximum principle of theorem 1 can be proved using the methods described by Derzko et al. [8] and Muzicant [23], where the optimal control problem is solved in a more general form.

The present value Hamiltonian for this problem is

$$
H=-e^{-r t} \rho(k-P)-e^{-r t} w+\lambda\left(-P_{a}-\bar{\mu} \Theta(P) \Psi(w) P\right),
$$

where $\lambda$ denotes the costate variable. The adjoint equation is given by

$$
\left.\frac{\partial \lambda}{\partial t}=-e^{-r t} \rho+\lambda \bar{\mu} \Psi(w)\left(P \Theta^{\prime}(P)+\Theta(P)\right)\right)-\frac{\partial \lambda}{\partial a} .
$$

Moving the last term $\partial \lambda / \partial a$ to the left hand side and substituting $\lambda=$ $e^{-r t} q^{*}$ yields equation (3.5). For calculating the maximum of the Hamiltonian 
$\max _{w} H$ all terms independent of $w(t, a)$ can be removed. So the remaining part is

$$
F(w):=-w-\bar{\mu} q^{*} P^{*} \Theta\left(P^{*}\right) \Psi(w) .
$$

The first and second order derivatives are (consider equation (2.9))

$$
\begin{aligned}
& F^{\prime}(w)=-1+(1-c) \varepsilon \bar{\mu} q^{*} P^{*} \Theta\left(P^{*}\right) e^{-\varepsilon w}, \\
& F^{\prime \prime}(w)=-(1-c) \varepsilon^{2} \bar{\mu} q^{*} P^{*} \Theta\left(P^{*}\right) e^{-\varepsilon w} .
\end{aligned}
$$

Solving $F^{\prime}(w)=0$ gives the optimal control of (3.7), and verifying that it is a maximum, we have to distinguish between two different cases:

1. $q^{*} \leq 0 \Rightarrow F^{\prime}(w)<0$, implying that $w^{*}=0$ is a maximum on the boundary, or

2. $q^{*}>0 \Rightarrow F^{\prime \prime}\left(w^{*}\right)<0$, implying that $w^{*}$ is a maximum in the interior.

\section{A.2 Proof for Theorem 2}

Brokate [4] has solved a more general control problem. So we can use those results to proof the theorem, but we have to do some transformations, because Brokate started from a minimizing instead of a maximizing problem.

Using our notation, the adjoint equations for the minimizing problem can be written as

$$
\begin{aligned}
\tilde{q}(t, a) & =\int_{t}^{T}-e^{-r \tau} \rho-\bar{\mu} \Phi\left(R_{\mathrm{soc}}^{*}(\tau, a)\right) \Psi\left(w^{*}(\tau, a)\right) \tilde{q}(\tau, a)-\frac{m_{2}}{k} \tilde{\theta}(\tau) d \tau, \\
\tilde{q}(T, a) & =0 \\
\tilde{\theta}(t) & =-\int_{0}^{\omega} \bar{\mu}(a) \Phi^{\prime}\left(R_{\mathrm{soc}}^{*}(t, a)\right) m_{1}(a) \Psi\left(w^{*}(t, a)\right) P^{*}(t, a) \tilde{q}(t, a) d a .
\end{aligned}
$$


Transforming the integral equation for $\tilde{q}$ into a differential equation and substituting $\tilde{q}=-e^{-r t} q$, we get equations (3.10) and (3.11).

For calculating the optimal control $w^{*}(t, a)$ it is necessary to maximize

$$
F(w):=-w-\bar{\mu} q^{*} P^{*} \Phi\left(m_{1} R_{\mathrm{soc}}^{*}\right) \Psi(w) .
$$

The same arguments as in the previous model hold here, too. So the optimal control of (3.13) maximizes the above function.

Remark. Applying this method of Brokate to the simplified model, where interaction takes place only between individuals of the same age, only slight changes of the proof are necessary to get the same results.

\section{References}

[1] D.A. Behrens, J.P. Caulkins, G. Tragler, and G. Feichtinger. Optimal control of drug epidemics: Prevent and treat — but not at the same time? Management Science, 46(3):333-347, 2000.

[2] D.A. Behrens, J.P. Caulkins, G. Tragler, and G. Feichtinger. Why present-oriented societies undergo cycles of drug epidemics. Journal of Economic Dynamics and Control, forthcoming.

[3] D.A. Behrens, J.P. Caulkins, G. Tragler, G. Feichtinger, and J.L. Haunschmied. A dynamic model of drug initiation: Implications for treatment and drug control. Mathematical Biosciences, 159:1-20, 1999.

[4] M. Brokate. Pontryagin's principle of control problems in age-dependent population dynamics. J. Math. Biology, 23:75-101, 1985. 
[5] S.N. Busenberg, M. Iannelli, and H.R. Thieme. Global behavior of an age-structured epidemic model. SIAM J. Math. Anal., 22(4):1065-1080, 1991.

[6] J.P. Caulkins, S. Paddock, R. Pacula, and J. Chiesa. School-Based Drug Prevention: What Kind of Drug Use Does it Prevent? MR-1459-RWJ. RAND, Santa Monica, CA, forthcomming 2002.

[7] J.P. Caulkins, C.P. Rydell, S.S. Everingham, J. Chiesa, and S. Bushway. An Ounce of Prevention, a Pound of Ucertainty: The Cost-Effectiveness of School-Based Drug Prevention Program. MR-923-RWJ. RAND, Santa Monica, CA, 1999.

[8] N. Derzko, S.P. Sethi, and G.L. Thompson. Distributed parameter systems approach to the optimal cattle ranching problem. Optimal Control Appl. Meth., 1:3-10, 1980.

[9] L.R.H. Drew. Drug dependence - a challenge to the a.c.t. Aust. J. Alcoholism Drug Dependence, 2:71-73, 1975.

[10] R.C. Engs. Cycles of social reform: Is the current anti-alcohol movement creating? Journal of Studies on Alcohol, 58(2):223-224, 1997.

[11] G. Feichtinger and R.F. Hartl. Optimale Kontrolle ökonomischer Prozesse. Walter de Gruyter, 1986.

[12] M. Iannelli, F.A. Milner, and A. Pugliese. Analytical and numerical results for the age-structured S-I-S epidemic model with mixed interintracohort transmission. SIAM J. Math. Anal., 23(3):662-688, 1992. 
[13] L.D. Johnston, P.M. O'Malley, and J.G. Bachman. Monitoring the Future National Survey Results on Drug Use, 1975-2000. Volume I: Secondary schooll students. (NIH Publication No. 01-4924). National Institute on Drug Abuse, Bethesda, MD, 2001.

[14] J. Kaplan. The Hardest Drug: Heroin and Public Policy. The University of Chicago Press, 1986.

[15] C.Y. Kaya, Y. Tugai, J.A. Filar, M.R. Agrawal, R.L. Ali, L.R. Gowing, and R. Cook. Heroin users in australia: Population trends. In submission.

[16] B.L. Keyfitz and N. Keyfitz. The McKendrick partial differential equation and its uses in epidomology and population studies. Math. Comput. Modelling, 26(6):1-9, 1997.

[17] M.A.R. Kleiman. Enforcement swamping: A positive feedback mechanism in rates of illicit activity. Math. Comput. Modelling, 27(2):67-95, 1993.

[18] J. Müller. Optimal vaccination patterns in age-structured populations. SIAM J. Appl. Math., 59(1):222-241, 1998.

[19] J. Müller. Optimal vaccination patterns in age-structured populations: Endemic case. Math. Comp. Modelling, 31(4-5):149-160, 2000.

[20] J.D. Murray. Mathematical Biology. Springer-Verlag, 2nd edition, 1993.

[21] D.F. Musto. The American Disease. Yale University Press, New Haven, CT, 1987. 
[22] D.F. Musto. Alcohol in american history. Scientific America, pages 78-83, April 1996.

[23] J. Muzicant. Systeme mit verteilten Parametern in der Bioökonomie Ein Maximumprinzip zur Kontrolle altersstrukturierter Modelle. PhD thesis, Vienna University of Technology, 1980.

[24] A. Preti, P. Miotto, and M. DeCoppi. Deaths by unintended illicit drug oversdose in italy, 1984-2000. Drug and Alcohol Dependence, 66:275-282, 2002 .

[25] W.J. Rorabaugh. The Alcoholic Republic: An American Tradition. Oxford University Press, 1979.

[26] W.E. Schiesser. The Numerical Method of Lines. Academic Press, 1991.

[27] G.W. Swan. Applications of Optimal Control Theory in Biomedicine. Dekker, 1984.

[28] M. Tamura. Japan: Stimulant epidemic past and present. Bulleting on Narcotics, 1:83-93, 1989. 


\section{Authors' biographies}

Christian Almeder is a Research Assistant in the Department of Operations Research and Systems Theory at the Vienna University of Technology. In 1999 he finished his Ph.D. on the dynamic modelling of the human arterial blood flow. In 2000 he was awarded by the President of the Austrian Republic with the promotio sub auspiciis praesidentis rei publicae for his studies. His research work appeared in several conference proceedings such as 2nd MathMod 1997, BMES \& EMBS 1999, EMBEC 1999, 3rd MathMod 2000, ASIM 2001, and others. His main research interests include modelling and simulation techniques, biomedical modelling, numerics of dynamical systems, and optimal control models with distributed parameters.

Jonathan P. Caulkins is Professor of Operations Research and Public Policy in the Heinz School of Public Policy and Managemant at Carnegie Mellon University. In 1990 he finished his Ph.D. on distribution and consumption of illicit drugs at M.I.T. From 1994 to 1996 he was codirector of RAND's Drug Policy Research Center and from 2000 to 2001 founding director of the Pittburgh Office of RAND. He has published articles in journals such as Journal of Economic Dynamics and Control, American Journal of Public Health, Journal of Policy Analysis and Management, Journal of Economics, Management Science, Operations Research, and Mathmatical and Computer Modelling. His current research interests are mathematical models of social policy problems, 
problems and policies concerning drugs, crime, and violence, and statistical rating and evaluation problems.

Gustav Feichtinger is Professor of Operations Research at the Vienna University of Technology and Director of the Department for Theoretical Demography at the Institute of Demography of the Austrian Academy of Sciences. He has written several books including: Optimale Kontrolle ökonomischer Prozesse: Anwendungen des Maximumprinzips in den Wirtschaftswissenschaften published by de Gruyter (with R.F. Hartl). His research work has appeared in Journal of Optimization Theory and Applications, Operations Research, Mathematical Biosciences, Optimal Control Applications and Methods, Mathematical Modelling of Systems, American Economic Review, International Journal of Game Theory, and others. His research interests include optimal control theory and dynamic games in economics and operations research, economics of crime, modelling of deviant behaviour, and demography.

Gernot Tragler is an Assistant Professor in the Department of Operations Research and Systems Theory at the Vienna University of Technology. For his Ph.D. from the Vienna University of Technology on the optimal control of illicit drug consumption, he received the annual award from the Austrian Society for Operations Research in 1999. His research work has appeared is such journals as Central European Journal for Operations Research and Economics, European Journal of Operational Research, International Game Theory Review, Journal of Economic Dynamics and Control, Journal of Economics, Journal of Opti- 
mization Theory and Applications, Management Science, Mathematical Biosciences, and Operations Research. His current research interests include deterministic, dynamic optimization with special emphasis on the control of illicit drug consumption, and cellular automata. 


\section{List of Figures}

1 Different types of initiation rates $\bar{\mu}(a)$ used in the simulation experiments. . . . . . . . . . . . . . 39

2 Influence $m\left(a, a^{\prime}\right)$ of the user group on the non-user group

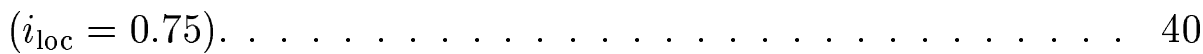

3 Change of the initiation rate depending on the reputation $\left(d_{1}=1, d_{2}=1, d_{3}=0.5,3\right) \ldots \ldots \ldots \ldots \ldots$

4 Comparison of the lifetime social costs depending on the initiation age with data from lifetime consumption of marijuana and cocaine........................ 42

$5 \quad P(t, a)$ in the case of a limit cycle $\left(d_{3}=3\right)$ using marijuana

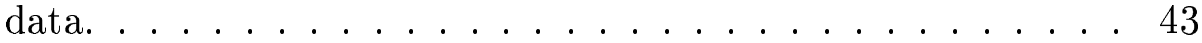

$6 \quad P(t, a)$ in the case of an equilibrium $\left(d_{3}=0.5\right)$ using marijuana

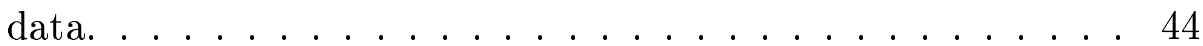

$7 \quad$ Optimal allocation of resources to prevention programs over different age classes over a time horizon of 200 years (using the marijuana data for the basic initiation rate). The middle region represent the stabilized situation. . . . . . . . . 45

8 Optimal allocation of resources to prevention programs over different age classes once the system has stabilized, e.g. $t=100$ years (using the marijuana data for the basic initiation rate, cf. figure 7$) \ldots \ldots \ldots \ldots \ldots$. . . . . . . . . 46 


\section{List of Tables}

1 Results of different simulation experiments. . . . . . . . . . 47

2 Comparison of results using optimal prevention and heuristic or no prevention. (For a description of the table entries see the footnotes of table 1$) \ldots \ldots \ldots 48$ 


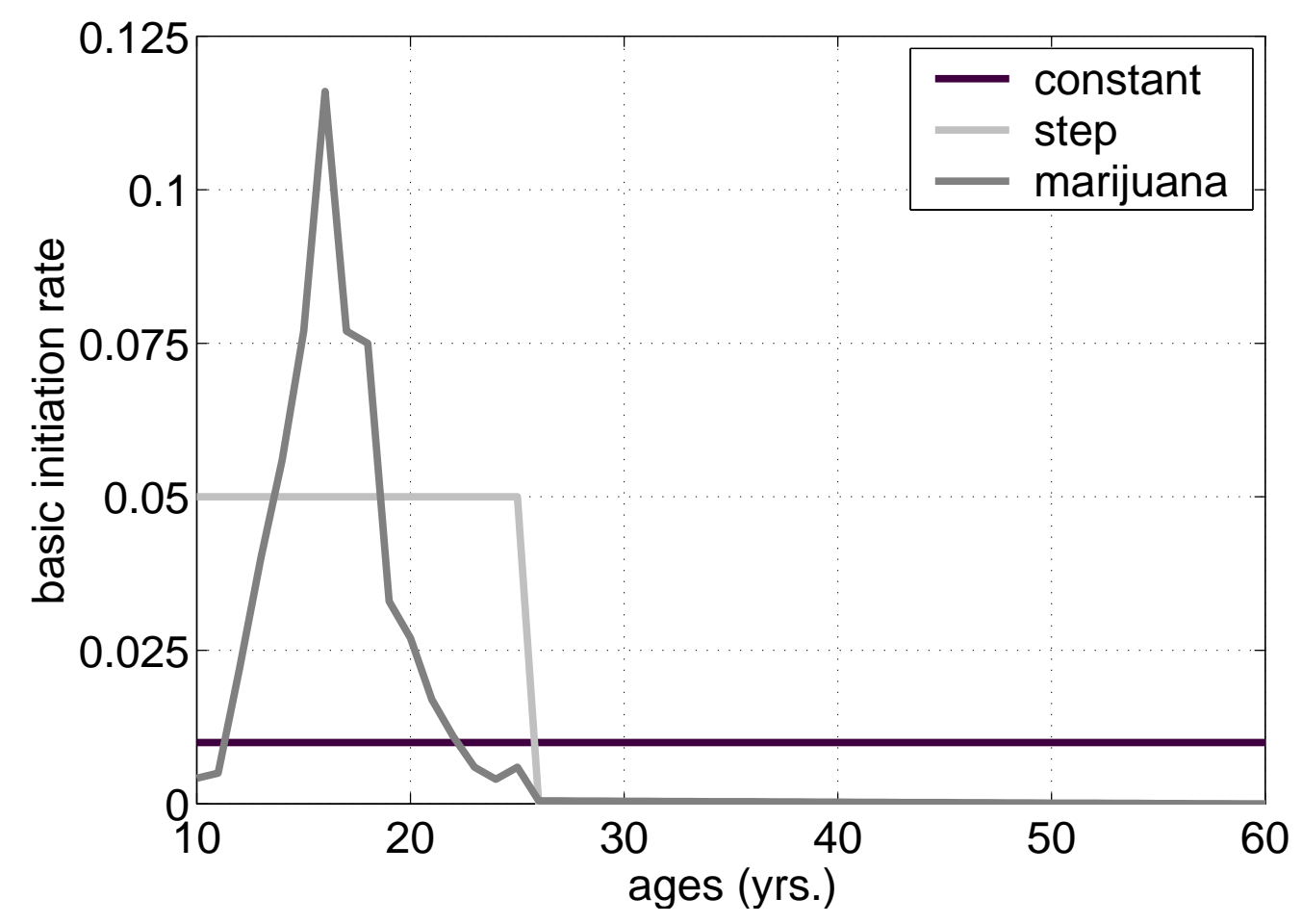

Figure 1: Different types of initiation rates $\bar{\mu}(a)$ used in the simulation experiments. 


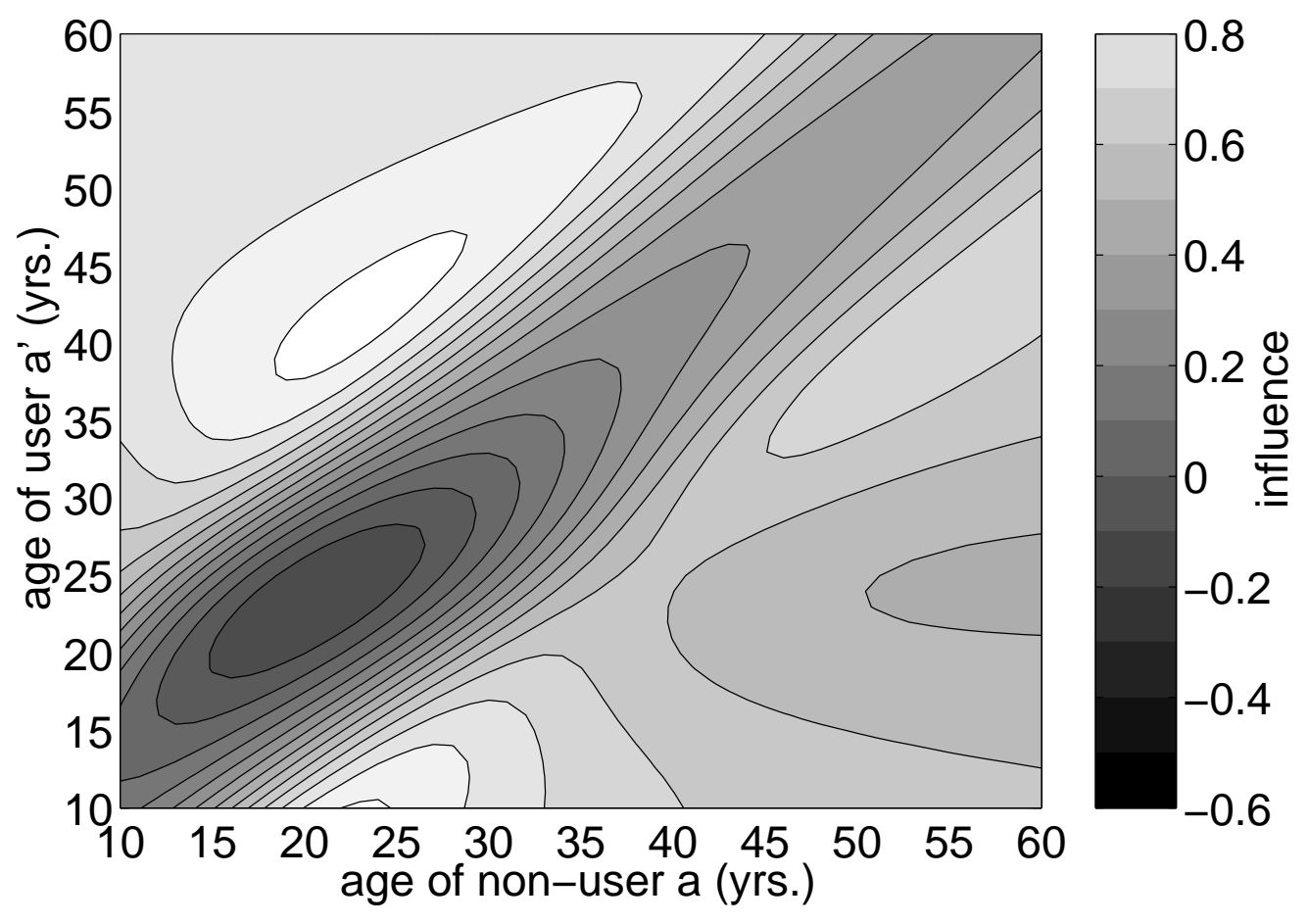

Figure 2: Influence $m\left(a, a^{\prime}\right)$ of the user group on the non-user group ( $i_{\text {loc }}=$ $0.75)$. 


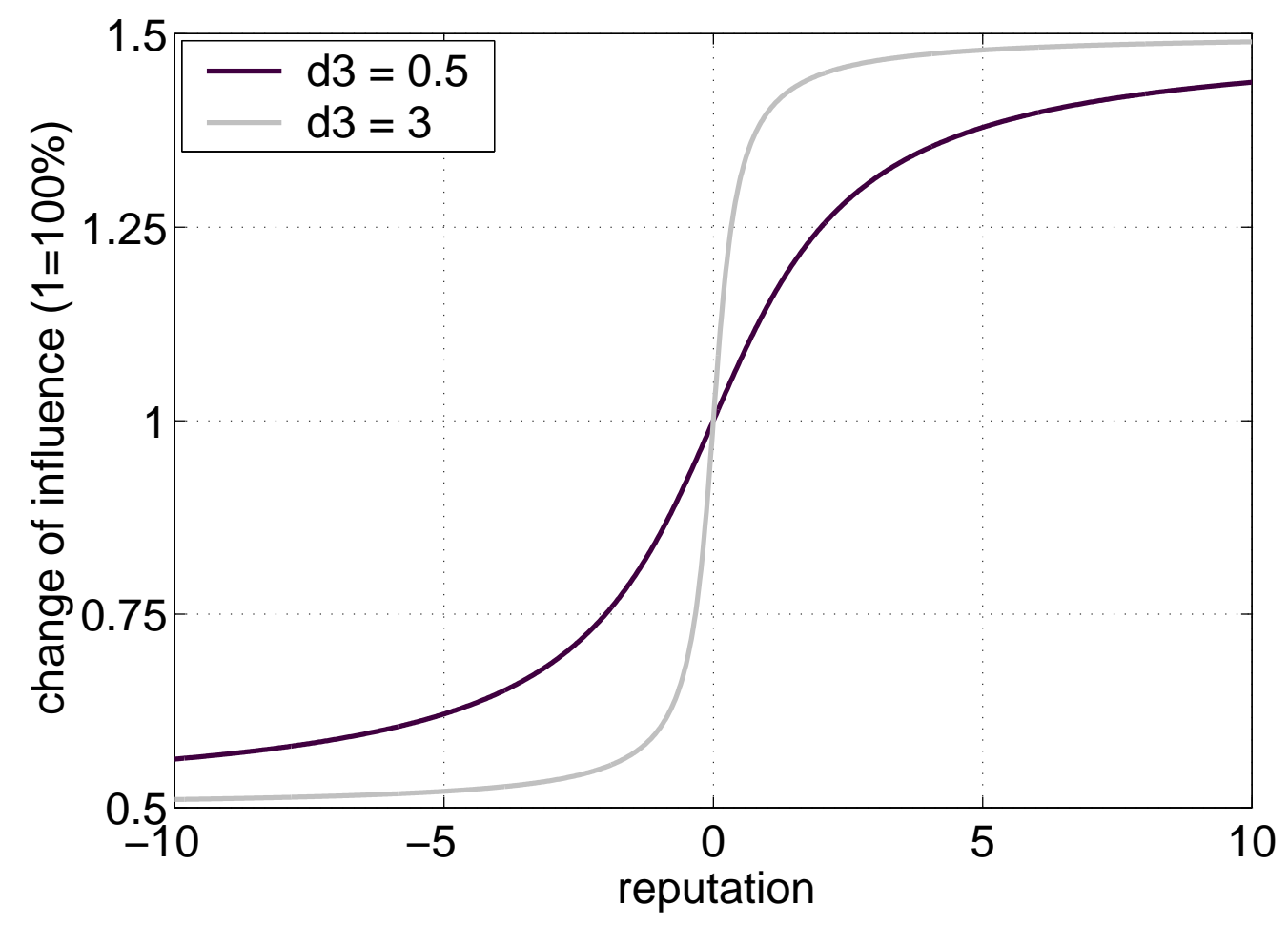

Figure 3: Change of the initiation rate depending on the reputation $\left(d_{1}=1\right.$, $\left.d_{2}=1, d_{3}=0.5,3\right)$. 


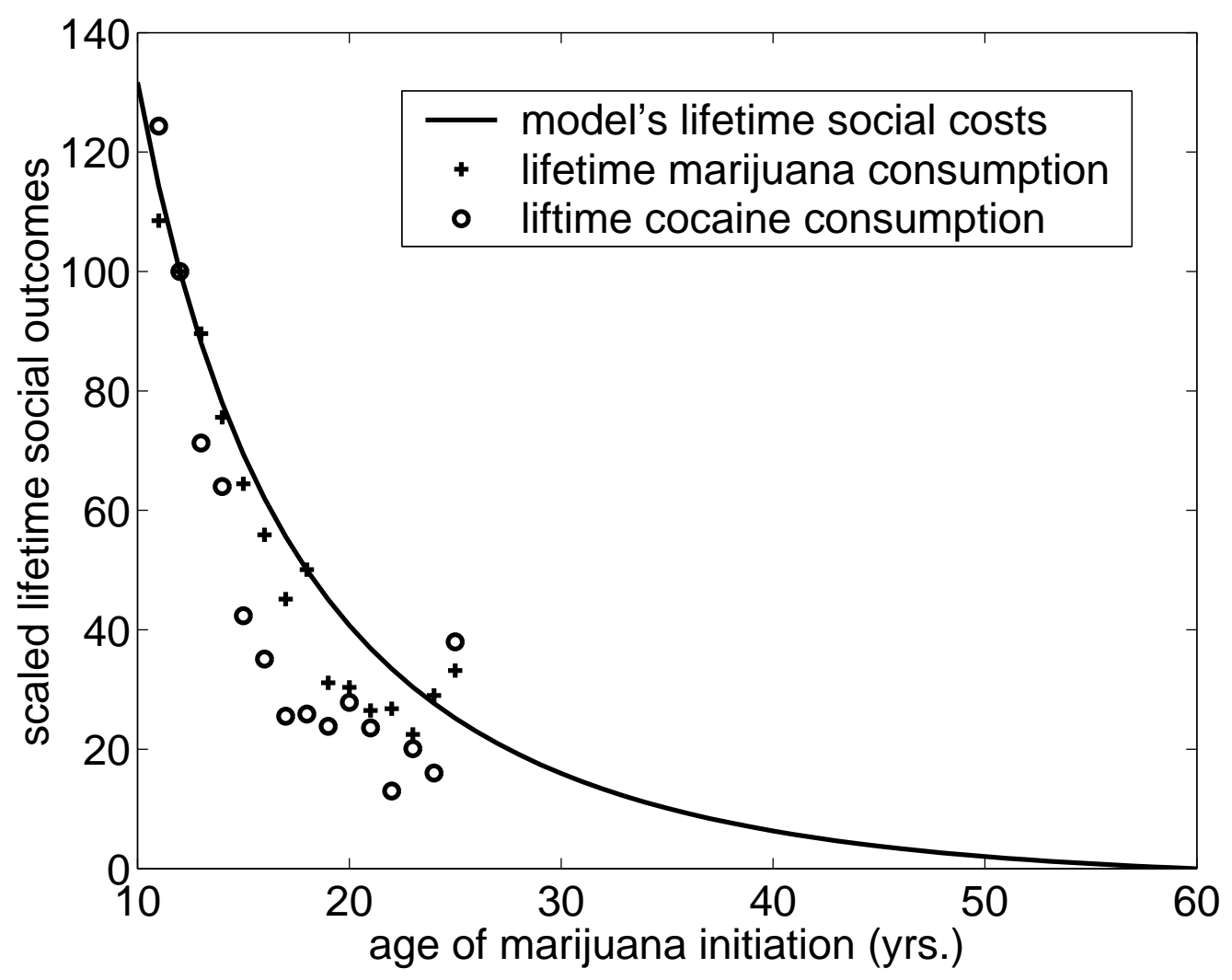

Figure 4: Comparison of the lifetime social costs depending on the initiation age with data from lifetime consumption of marijuana and cocaine. 


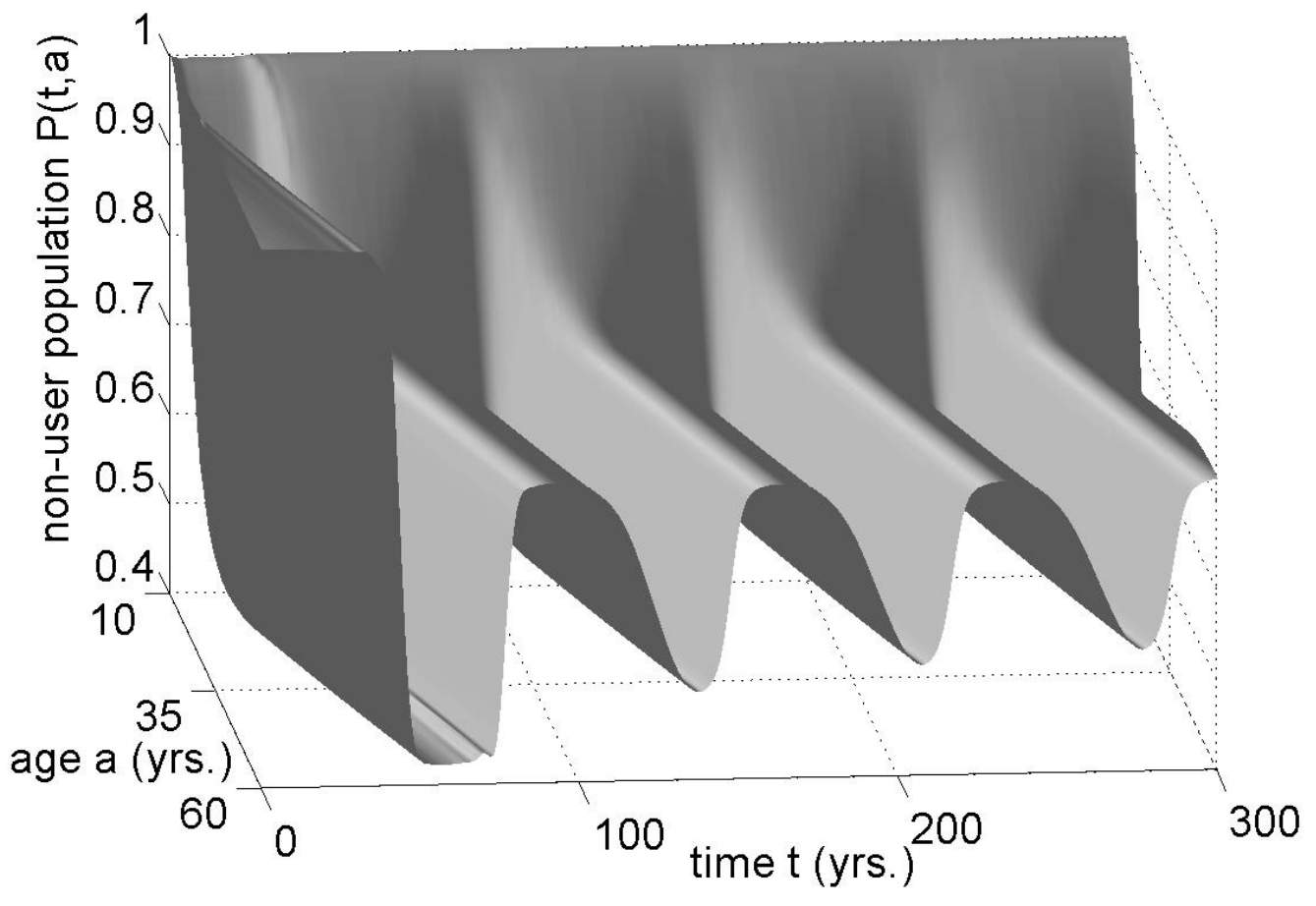

Figure 5: $P(t, a)$ in the case of a limit cycle $\left(d_{3}=3\right)$ using marijuana data. 


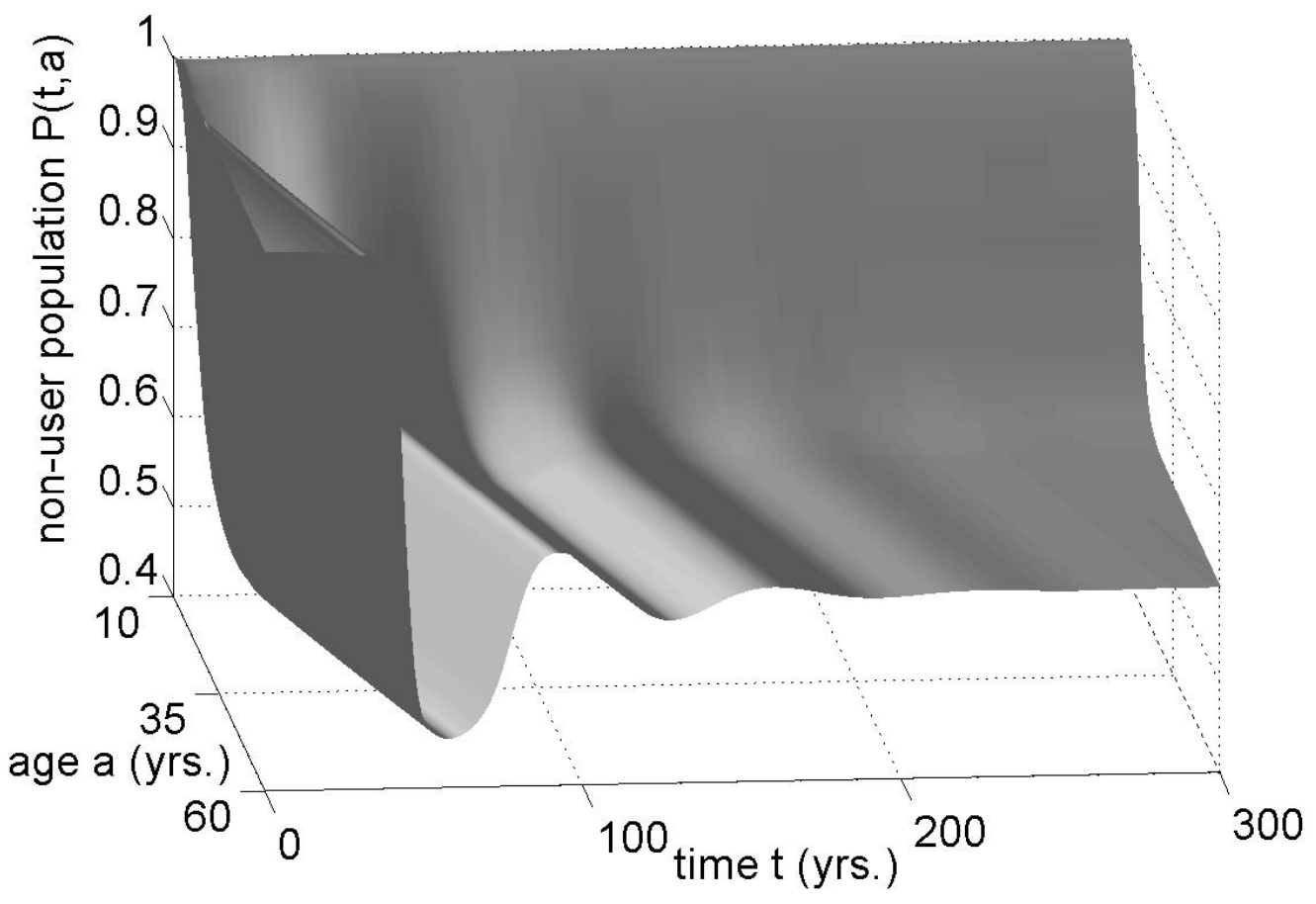

Figure 6: $P(t, a)$ in the case of an equilibrium $\left(d_{3}=0.5\right)$ using marijuana data. 


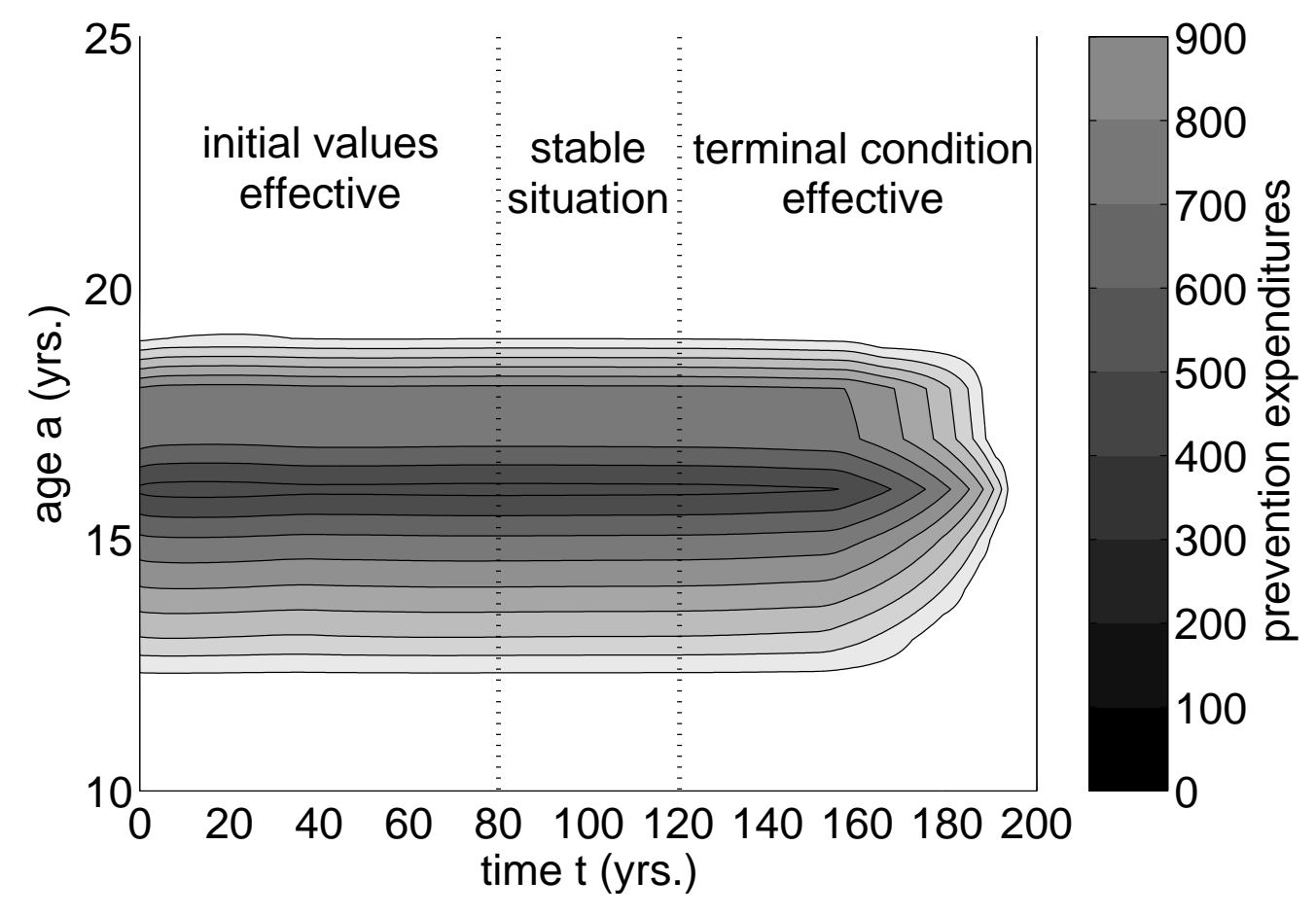

Figure 7: Optimal allocation of resources to prevention programs over different age classes over a time horizon of 200 years (using the marijuana data for the basic initiation rate). The middle region represent the stabilized situation. 


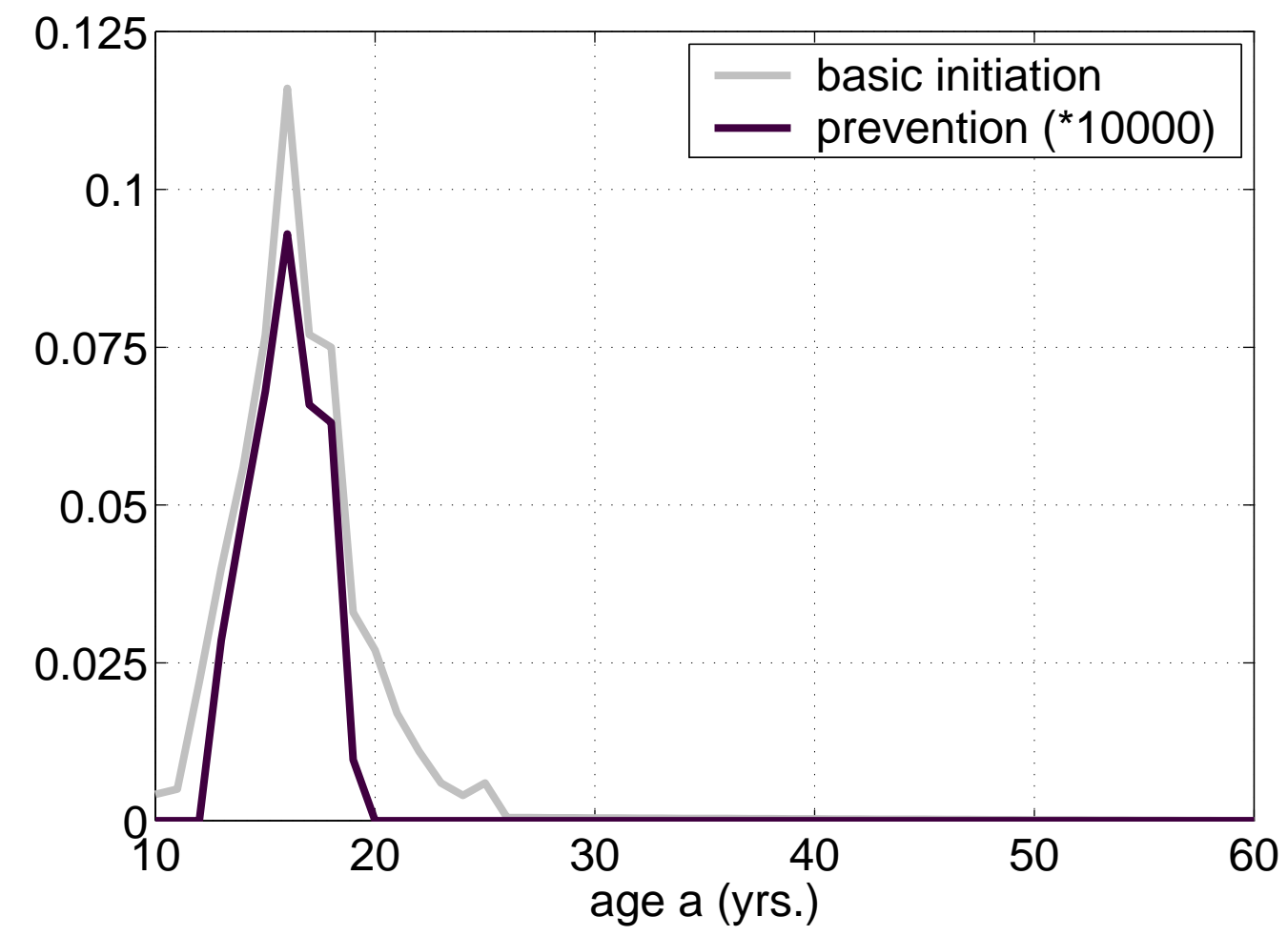

Figure 8: Optimal allocation of resources to prevention programs over different age classes once the system has stabilized, e.g. $t=100$ years (using the marijuana data for the basic initiation rate, cf. figure 7). 


\begin{tabular}{c|c|c||c|c|c|c|c}
\multicolumn{9}{c||}{ Model parameters } & \multicolumn{7}{c}{ Results } \\
$\bar{\mu}(a)^{1}$ & $d_{3}$ & $w(t, a)^{2}$ & $\mathrm{LC}^{3}$ & $\mathrm{U}^{4}$ & $\mathrm{MI}^{5}$ & $\mathrm{aMI}^{6}$ & $\mathrm{TSC}^{7}$ \\
\hline \hline const. & 0.5 & 0 & 0 & 0.37 & 0.0077 & 43 & 0.58 \\
\hline const. & 3 & 0 & 0 & 0.34 & 0.0095 & 47 & 0.55 \\
\hline step & 0.5 & 0 & 0 & 0.49 & 0.033 & 10 & 1.22 \\
\hline step & 3 & 0 & 62.5 & $0.36-0.51$ & 0.043 & 21 & 1.26 \\
\hline mari. & 0.5 & 0 & 0 & 0.39 & 0.078 & 16 & 0.96 \\
\hline mari. & 3 & 0 & 71.4 & $0.28-0.46$ & 0.096 & 16 & 1.00 \\
\hline mari. & 0.5 & step & 0 & 0.38 & 0.075 & 16 & 0.94 \\
\hline mari. & 3 & step & 71.4 & $0.26-0.44$ & 0.093 & 16 & 0.98 \\
\hline mari. & 0.5 & init & 0 & 0.37 & 0.071 & 16 & 0.92 \\
\hline mari. & 3 & init & 71.4 & $0.26-0.42$ & 0.081 & 16 & 0.96
\end{tabular}

1 Three different forms for the basic initiation rates $\bar{\mu}(a)$ are used (const. - constant; step - constant between 0 and 25, otherwise 0; mari. marijuana initiation; cf. figure 1).

2 For the prevention $w(t, a)$ we tested three different cases, which are all independent of $t(0$ - no prevention; step - constant between 10 to 25 , otherwise 0 ; init - prevention expenditure proportional to basic initiation rate between 10 and 25). The step and init forms are chosen to have the same total prevention expenditure.

3 LC - limit cycle for the last age class (60 yrs.). The value in this column indicates the duration of the cycles (in years). If the system converges to an equilibrium, the entry is 0 .

$4 \mathrm{U} 60$ - the proportion of people who try the drug at some point in their life.

$5 \mathrm{MI}$ - the maximal incidence rate.

6 aMI - age of maximal incidence rate.

7 TSC - total social costs according to equation (2.10) relative to the results in the 6 th row(marijuana data, $d_{3}=3$, no prevention). The effectiveness of prevention programs w.r.t. the total social costs depends on the amount of social costs assumed to be generated by one drug user per year.

Table 1: Results of different simulation experiments. 


\begin{tabular}{c||c|c|c|c}
$w(t, a)$ & $\mathrm{U} 60$ & $\mathrm{MI}$ & $\mathrm{aMI}$ & $\mathrm{TSC}$ \\
\hline \hline 0 & 0.29 & 0.057 & 16 & 1.000 \\
\hline step & 0.28 & 0.055 & 16 & 0.993 \\
\hline init & 0.27 & 0.052 & 16 & 0.983 \\
\hline optimal & 0.27 & 0.051 & 16 & 0.981
\end{tabular}

Table 2: Comparison of results using optimal prevention and heuristic or no prevention. (For a description of the table entries see the footnotes of table 1) 